\title{
Comparison of cardiolipin and treponemal tests in the serodiagnosis of yaws
}

\author{
H. E. MENKE,* J. VELDKAMP,* E. A. BRUNINGS, $†$ P. L. A. NIEMEL, \\ A. NOTOWICZ§ AND E. STOLZ§ \\ From the *National Institute of Public Health, Bilthoven, The Netherlands; the †Professor P. C. Flu \\ Institute and the $\ddagger$ Dermatological Service, Paramaribo, Surinam; and the §Department of Dermatology, \\ University Hospital, Rotterdam (Dijkzigt), The Netherlands
}

SUMMARY Results of the Venereal Disease Research Laboratory (VDRL), rapid plasma reagin (RPR), Treponema pallidum haemagglutination (TPHA), T. pallidum immobilisation (TPI), and fluorescent treponemal antibody absorption (FTA-ABS) tests on sera of 661 children from a region where yaws is hypoendemic are compared. For $107(16.2 \%)$ out of 661 sera the FTA-ABS test was the only one showing reactivity; in these instances the test was weakly reactive (intensity of fluorescence scored as + ) and the children had no history and no signs or symptoms of treponemal disease. A solitary, weakly reactive FTA-ABS test result seems to have no clinical significance in these cases. The FTA-ABS test can be used as a confirmatory test for yaws instead of the TPI test, if only the results of sera showing an intensity of fluorescence scored as ++ or more are considered to be positive. There appeared to be no significant differences in the results of the VDRL, RPR, and TPHA tests as screening tests for yaws when the TPI or FTA-ABS tests were used as reference tests.

\section{Introduction}

The value of various serological tests for the screening and confirmation of syphilis has been fairly well established. The literature on their use for screening and diagnosis of other treponematoses, however, is scanty. This study was carried out to assess the value of cardiolipin and treponemal tests in the serodiagnosis of yaws.

\section{Material and methods}

In this study, carried out in 1976 and 1977, initially a total of 969 children living in an area of Surinam where yaws is hypoendemic were examined; clinical and epidemiological data on these children have been given (Niemel et al., 1979). Blood samples from all the children were collected by venepuncture. A part of each serum sample was used in Surinam for the Venereal Disease Research Laboratory (VDRL) test and the remainder was stored at $-70^{\circ} \mathrm{C}$ before being sent to the Netherlands for

Address for reprints: Dr H. E. Menke, National Institute of Public Health, PO Box 1, Bilthoven, The Netherlands

Received for publication 3 October 1978 other tests. The VDRL slide test was performed at the laboratory of the Professor P. C. Flu Institute, Paramaribo, Surinam, according to the recommended procedure (National Communicable Disease Center, 1969). Reagents were purchased from Baltimore Biological Laboratories (Cockeysville, Maryland 21030, USA).

The rapid plasma reagin (RPR) card test and the Treponema pallidum haemagglutination (TPHA) test were performed at the laboratory of the dermatology department, University Hospital, Rotterdam-Dijkzigt, according to manufacturer's manual. RPR kits were purchased from Hynson, Westcott and Dunning Inc. (Baltimore, Maryland 21201, USA). Reagents for the TPHA test (manufactured by Wellcome Reagents Ltd, Beckenham, Kent) were obtained from Wellcome Nederland BV, Amsterdam.

The $T$. pallidum immobilisation (TPI) test and the fluorescent treponemal antibody absorption (FTA-ABS) test were performed at the venereal disease department of the bacteriology laboratory, National Institute of Public Health, Bilthoven, The Netherlands. Both tests were performed according to the recommended procedures (National Communicable Disease Center, 1969) with slight modi- 
fications; in the TPI test lysozyme $(100 \mu \mathrm{g} / \mathrm{ml})$ was added to the sustaining medium; in the FTA-ABS test the sera were used in dilutions of $1 / 12$ instead of $1 / 5$ after absorption with an ultrasonicate of Reiter treponemes instead of sorbent.

The results of the VDRL, RPR, TPHA, and TPI tests were scored as either positive or negative. The result of the FTA-ABS test was scored as positive when the intensity of fluorescence was ++ or more, weakly positive when the intensity of fluorescence was + , or negative.

For comparing the results of the screening tests (a) (VDRL, RPR, and TPHA tests), the TPI test and FTA-ABS test were used as reference tests(b). The criteria used in this comparison are defined as follows:

(1) Agreement $=$

number of sera giving identical* results in (a) and (b) total number of sera investigated

(2) Sensitivity=

number of sera positive in (a) and (b)

total number of sera positive in (b)

(3) Rate of possible false-positive reactions= number of sera positive in (a) and negative in (b)

$$
\text { total number of sera investigated }
$$

*identical means either positive or negative

For statistical analysis, the $\chi^{2}$ test for randomised block designs with data arranged in two classes was used.

\section{Results}

When samples arrived in the Netherlands, 308 of 969 samples contained too little serum for carrying out the RPR, TPHA, TPI, and FTA-ABS tests; these samples were, therefore, excluded from this study.

Of the remaining 661 serum samples, the VDRL test gave positive results in 131 ; of these 131 patients, seven had primary yaws, eight secondary yaws, and 116 latent yaws.

Table 1 shows the reactivity of the various serological tests on the 661 samples. The FTA-ABS test was reactive for 261 samples; for 154 of these the result was positive and for 107 weakly positive. For these 107 cases, the FTA-ABS test was the only test giving a reactive result. The 107 patients showed no signs or symptoms and gave no history of treponemal disease. All tests were reactive for the 15 cases of early infectious yaws.

In Table 2 the results of the FTA-ABS and TPI tests are compared. The agreement between the
Table 1 Reactivity of various tests on sera from 661 children from a region of hypoendemic yaws infection

\begin{tabular}{llll}
\hline \multirow{2}{*}{ Test } & \multicolumn{2}{l}{ Sera with positive results } & \\
\cline { 2 - 3 } & No. & $\%$ & $\begin{array}{l}\text { No. of sera with } \\
\text { negative results }\end{array}$ \\
\hline VDRL & 131 & $19 \cdot 8$ & 530 \\
RPR & 127 & $19 \cdot 2$ & 534 \\
TPHA & 139 & $21 \cdot 0$ & 522 \\
TPI & 147 & $22 \cdot 1$ & 514 \\
FTA-ABS & $261^{*}$ & $39 \cdot 5$ & 400 \\
\hline
\end{tabular}

*107 sera with weakly positive results included

Table 2 Comparison of results of FTA-ABS and TPI tests

\begin{tabular}{llll}
\hline \multirow{4}{*}{ TPI test results } & \multicolumn{3}{l}{ FTA-ABS test results } \\
\cline { 2 - 4 } & Negative & Positive* & Total \\
\hline Negative & 396 & 118 & 514 \\
Positive & 4 & 143 & 147 \\
Total & 400 & 261 & 661 \\
\hline
\end{tabular}

*107 weakly positive results to FTA-ABS tests (all negative in TPI test) included

results of FTA-ABS test and TPI test, calculated from the figures presented in this Table, is $81.5 \%$. If the 107 sera with a weakly positive result in the FTA-ABS test are not regarded as giving positive results, the agreement between the FTA-ABS and TPI tests is $97.3 \%$.

The results of the VDRL, RPR, and TPHA tests (screening tests), when the TPI test is used as a reference, are compared in Table 3. There appear to be no statistically significant differences between the three screening tests when agreement, sensitivity, and rate of possible false-positive reactions are taken as criteria. If the FTA-ABS test is used as the reference test instead of the TPI test (Table 2) again no statistically significant differences are found between the three screening tests (level of significance, $5 \%$ ).

Table 3 Comparison of results of VDRL, RPR, and TPHA test on 661 sera when either the TPI test or the $F T A-A B S$ test * was used as reference. (Figures for the FTA-ABS test used as a reference are given in brackets)

\begin{tabular}{|c|c|c|c|c|c|c|}
\hline \multirow{3}{*}{$\begin{array}{l}\text { Agreement } \\
\text { Sensitivity } \\
\text { Rate of possible } \\
\text { false-positive }\end{array}$} & \multicolumn{2}{|c|}{$\begin{array}{l}\text { VDRL test } \\
(\%)\end{array}$} & \multicolumn{2}{|c|}{$\begin{array}{l}R P R \text { test } \\
(\%)\end{array}$} & \multicolumn{2}{|c|}{$\begin{array}{l}\text { TPHA test } \\
(\%)\end{array}$} \\
\hline & $\begin{array}{l}93.0 \\
79.6\end{array}$ & $\begin{array}{l}(94 \cdot 1) \\
(79 \cdot 9)\end{array}$ & $\begin{array}{l}94 \cdot 3 \\
80 \cdot 2\end{array}$ & $\begin{array}{l}(95 \cdot 0) \\
(80 \cdot 5)\end{array}$ & $\begin{array}{l}94.6 \\
85.0\end{array}$ & $\begin{array}{l}(96 \cdot 2) \\
(87 \cdot 0)\end{array}$ \\
\hline & $2 \cdot 1$ & $(1 \cdot 2)$ & 1.4 & $(0.5)$ & $2 \cdot 1$ & $(0.8)$ \\
\hline
\end{tabular}

*In these calculations only the results of sera showing an intensity of fluorescence scored as ++ or more in the FTA-ABS test are considered as reactive 


\section{Discussion}

A single, weakly reactive FTA-ABS result in the absence of a history and clinical signs or symptoms of treponemal disease was found for $107(16.2 \%)$ of 661 sera. Garner et al. (1970) reported a similar finding for $4.6 \%$ of 848 sera from an area of yaws infection. In 10 of our cases new serum samples were collected after one year and the FTA-ABS test was still weakly positive and was the only test showing reactivity.

This indicates that a single, weakly reactive result to the FTA-ABS test is not necessarily the first sign of an infection with pathogenic treponemes. The cause of a single, weakly reactive result is not known; several hypotheses, however, can be put forward:

(1) Individuals with a single, weakly reactive result to the FTA-ABS test may have been infected by pathogenic treponemes; they may not, however, have developed clinical signs of disease nor an extensive humoral immunological response.

(2) Not all non-specific antibodies may have been removed from the serum with our ultrasonicate. The weak fluorescence might have disappeared if an ultrasonicate from other non-pathogenic treponemes as well as Reiter treponemes had been used.

(3) The single, weakly reactive result to the FTA-ABS test may have been caused by other (non-treponemal) diseases and should be considered as a biological false-positive reaction.

Whatever the cause, the single, weakly reactive result does not indicate active treponemal disease and seems to have few practical consequences.

The TPI test is generally accepted as a test for confirmation of treponemal disease. If only those sera which gave results of ++ or more in the FTA-ABS test are considered as reactive, the agreement between the FTA-ABS test and the TPI test appears to be $97 \cdot 3 \%$. Thus, the FTA-ABS test can also be used as a confirmatory test for yaws, if only the results of sera showing an intensity of fluorescence scored as ++ or more are considered as reactive.

There appear to be no significant differences between the VDRL, RPR, and TPHA tests-all of which are generally recommended for screeningwhen agreement with either TPI or FTA-ABS tests, sensitivity, and rate of possible false-positive reactions are taken as criteria. Thus, other criteria, such as cost and labour, should be taken into account when one of these screening tests is selected for future use.

Although the figures in Table 3 suggest that the TPHA test is more sensitive than the other two screening tests, the differences in sensitivity are not statistically significant. According to MacFarlane and Elias-Jones (1977), the sensitivity of the Wellcome TPHA test appears to increase if this test is performed at a lower screening dilution than recommended by the manufacturer. Our results, therefore, might have been different if the TPHA test had been performed as suggested by these authors.

We thank Mr A. B. Leussink of the Department of Biometrical Analysis, the National Institute of Public Health, Bilthoven, for his help. TPHA-test reagents were kindly supplied by Wellcome Nederland BV, Amsterdam.

\section{References}

Garner, M. F., Backhouse, J. L., Cook, C. A., and Roeder, P. J. (1970). Fluorescent treponemal antibody absorption (FTA-ABS) test in yaws. British Journal of Venereal Diseases, 46, 284-286.

MacFarlane, D. E., and Elias-Jones, T. F. (1977). Screening tests for syphilis. A comparison of the Treponema pallidum haemagglutination assay with two automated serological tests. British Journal of Venereal Diseases, 53, 348-352.

National Communicable Disease Center (1969). Manual of Tests for Syphilis. USPHS Publication No. 411. US Printing Office: Washington DC.

Niemel, P. L. A., Brunings, E. and Menke, H. E. (1979). Attenuated yaws in Surinam. British Journal of Venereal Diseases, 55, 99-101. 